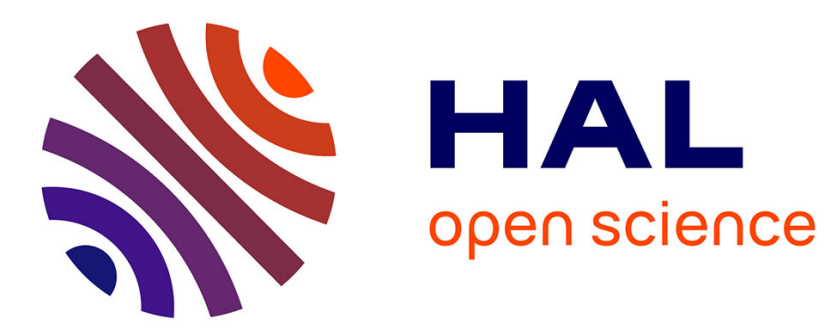

\title{
Geometric and elastostatic calibration of robotic manipulator using partial pose measurements
}

Alexandr Klimchik, Yier Wu, Stéphane Caro, Benoit Furet, Anatol Pashkevich

\section{To cite this version:}

Alexandr Klimchik, Yier Wu, Stéphane Caro, Benoit Furet, Anatol Pashkevich. Geometric and elastostatic calibration of robotic manipulator using partial pose measurements. Advanced Robotics, 2014, 28, pp.1419 - 1429. 10.1080/01691864.2014.955824 . hal-01084653

\section{HAL Id: hal-01084653 https://hal.science/hal-01084653}

Submitted on 19 Nov 2014

HAL is a multi-disciplinary open access archive for the deposit and dissemination of scientific research documents, whether they are published or not. The documents may come from teaching and research institutions in France or abroad, or from public or private research centers.
L'archive ouverte pluridisciplinaire HAL, est destinée au dépôt et à la diffusion de documents scientifiques de niveau recherche, publiés ou non, émanant des établissements d'enseignement et de recherche français ou étrangers, des laboratoires publics ou privés. 


\title{
FULL PAPER
}

\section{Geometric and elastostatic calibration of robotic manipulator using partial pose measurements}

\author{
Alexandr Klimchik ${ }^{a, b *}$, Yier $\mathrm{Wu}^{a, b}$, Stephane Caro $^{b, c}$, Benoit Furet ${ }^{b, d}$ and Anatol Pashkevich ${ }^{a, b}$ \\ ${ }^{a}$ Ecole des Mines de Nantes (EMN), 4 rue Alfred-Kastler, Nantes 44307, France; \\ ${ }^{b}$ Institut de Recherches en Communications et en Cyberntique de Nantes (IRCCyN), \\ UMR CNRS 6597, 1 rue de la Noe, 44321 Nantes, France; \\ ${ }^{c}$ Centre national de la recherche scientifique (CNRS), France; \\ ${ }^{d}$ Universit de Nantes: Institut Universitaire de Technologie (IUT) de Nantes, \\ 2 avenue du Professeur Jean Rouxel, 44475 Carquefou, France;
}

(v1.0 released September 2013$)$

\begin{abstract}
The paper deals with geometric and elastostatic calibration of robotic manipulator using partial pose measurements, which do not provide the end-effector orientation. The main attention is paid to the efficiency improvement of identification procedure. In contrast to previous works, the developed calibration technique is based on the direct measurements only. To improve the identification accuracy, it is proposed to use several reference points for each manipulator configuration. This allows avoiding the problem of non-homogeneity of the least-square objective, which arises in the classical identification technique with the full-pose information (position and orientation). Its efficiency is confirmed by the comparison analysis, which deals with the accuracy evaluation of different identification strategies. The obtained theoretical results have been successfully applied to the geometric and elastostatic calibration of serial industrial robot employed in a machining work-cell for aerospace industry.
\end{abstract}

Keywords: robot calibration; parameter identification; partial pose measurement; accuracy improvement

\section{Introduction}

To achieve the desired accuracy, each industrial robot must go through the calibration procedure, which deals with proper parameter tuning of the mathematical model embedded in the robot controller $[1,2]$. Because of its importance, the problem of robot calibration has been in the focus of research community for many years and has been studied from different aspects [3-12]. In spite of this, the issues of the identification accuracy and calibration error reduction have not found enough attention, only limited number of works directly addressed these important problems [13]. Generally, two main approaches that allow us to improve the identification accuracy without increasing the number of experiments exist. The first one deals with the preliminary optimization of the manipulator measurement configurations (so-called design of calibration experiments). The second approach consists in enhancing the objective function to be minimized inside the identification algorithm (in order to minimize impact of the measurement noise). As follows from the literature analysis, the first approach has been considered in a number of papers [14-18], while the second one received less attention of the researches. For this reason, taking into account particularities of the measurement system used in our experiments, this paper focuses on the improvement of the second method, which looks rather promising here.

In robot calibration, there exists a number of techniques that differ in the measurement equipment, the nature of the experimental data (position, orientation, distance, etc.) and in the opti- 
mization algorithm that produces the desired parameters [19-28]. At present, the most popular are the so-called open-loop methods that utilize external measurement devices to obtain either a full or partial pose ${ }^{1}$ of the end-effector (i.e. position and/or orientation) [22-28]. However, it should be noted that the manipulator end-effector orientation cannot be measured directly, so the orientation angles are calculated using positions of several points around the end-effector center point (TCP). A relevant example can be found in [29], where the end-effector orientation is evaluated using three target points located on the special measurement flange (i.e., three orientation angles are computed from nine Cartesian coordinates provided by the laser tracker). However this approach, which is based on the minimization of the squared sum of the position and orientation residuals, does not allow to minimize the measurement errors impact in the best way (in fact, the position and orientation components of the objective to be minimized are not weighted properly from the statistical point of view). To overcome this difficulty, in this paper it is proposed to use only direct measurement information, i.e. to replace the conventional objective function (squared sum of the position and orientation residuals) by a homogeneous squared sum of position residuals for all measurement points. In contrast to previous works that also based on the partial pose measurements, here it is proposed to use the same data as for the full-pose approach (i.e. several reference points for each configuration in order to compute orientations), but using all direct measurements only without computing the orientation angles. It is clear that this approach is promising for the identification accuracy improvement, but it requires some revisions of the identification algorithm, which is proposed in this paper.

The main contribution of the paper is enhancing partial pose measurement technique. It is proposed to use Cartesian coordinate measurements for several reference points located on the end-effector and to avoid direct computation of the end-effector orientation. It is worth mentioning that usual techniques implement other ideas: (i) computation full pose coordinates (position and orientation) from three reference points that are directly included in the identification equations; (ii) using Cartesian coordinates of a single reference point obtained for exhaustive number of measurement configurations. Compared to previous works, the proposed approach has essential advantages. In particular, in the case of three reference points (minimum to compute full pose coordinate), the new approach will have 9 identification equations instead of 6 for each manipulator configuration. However, this approach requires additional parameters that define tool transformation for each reference point. Therefore, in this paper a particular attention is paid to the developing of the mathematical routines for simultaneous parameters identification for several tool and base transformations, which correspond to different reference points and measurement system locations. In contrast to other works, such an approach can be efficiently applied both for elastostatic and geometric calibration and allows user to obtain not the manipulator parameters only but also the base and tool transformations.

To address the above defined problem, the remainder of this paper is organized as follows. Section 2 defines the research issue and basic assumptions. In Sections 3, the identification algorithm is presented. Section 4 proposes comparison of the developed identification algorithm with the conventional one. Section 5 presents an application example illustrating benefits of the proposed approach. Finally, Section 6 summarizes the main contributions of the paper.

\section{Problem statement}

Let us consider a serial robot whose end-effector location $\boldsymbol{t}=(\boldsymbol{p}, \boldsymbol{\varphi})$ (position $\boldsymbol{p}$ and orientation $\varphi$ ) is computed using the following vector function

$$
\boldsymbol{t}=g(\boldsymbol{q}, \boldsymbol{\theta}, \boldsymbol{\pi})
$$

\footnotetext{
${ }^{1}$ The term "partial pose" is adopted here to stress that only positional components of the "full pose" (position and orientation) is used. This terminology has been also previously used by other authors [9].
} 
where $g($.$) defines the manipulator extended geometric model, \boldsymbol{q}$ is the vector of actuated coordinates, $\boldsymbol{\theta}$ is the vector of robot elastostatic deflections, and the vector of the parameters $\boldsymbol{\pi}=\boldsymbol{\pi}_{0}+\Delta \boldsymbol{\pi}$ is presented as the sum of the nominal component $\boldsymbol{\pi}_{0}$ and geometrical errors $\Delta \boldsymbol{\pi}$ to be identified via calibration.

In addition to the geometric equation (1), let us consider the elastostatic model that allows us to compute the deflections $\boldsymbol{\theta}$ caused by the external loading $\boldsymbol{F}$ applied to the manipulator end-effector. It should be mentioned that taking into account the elastostatic deflections is very important in many industrial applications (robotic-based milling, friction stir welding, etc.), where essential forces caused by the manufacturing task are applied to the robot end-effector [30]. In this case, the compliance errors become the main source of the manipulator inaccuracy and should be compensated using either on-line or off-line compensation technique [31]. The relevant equation that allows us to compute deflections in virtual joints (or in actuated joints if their suppleness is a major source of the manipulator elasticity) can be presented in the following form $[32]$

$$
\boldsymbol{\theta}=\boldsymbol{k}_{\theta} \cdot \boldsymbol{J}_{\theta}^{\mathrm{T}} \cdot \boldsymbol{F}
$$

where the matrix $\boldsymbol{J}_{\theta}=\partial g(\boldsymbol{q}, \boldsymbol{\theta}, \boldsymbol{\pi}) / \partial \boldsymbol{\theta}$ is the manipulator Jacobian with respect to the elastostatic deflections $\boldsymbol{\theta}$, and $\boldsymbol{k}_{\theta}$ is the manipulator compliance matrix to be identified.

Assuming that the values $\Delta \boldsymbol{\pi}$ and $\boldsymbol{\theta}$ are relatively small, equation (1) can be linearized and presented in the form

$$
\boldsymbol{t}=\boldsymbol{g}_{0}+\boldsymbol{J}_{\pi} \cdot \Delta \boldsymbol{\pi}+\boldsymbol{J}_{\theta} \cdot \boldsymbol{k}_{\theta} \cdot \boldsymbol{J}_{\theta}^{T} \cdot \boldsymbol{F}
$$

where the first term $\boldsymbol{g}_{0}=g\left(\boldsymbol{q}_{i}, \mathbf{0}, \boldsymbol{\pi}_{0}\right)$ corresponds to the nominal geometric model (i.e. to the case when $\boldsymbol{\theta}=\mathbf{0}, \Delta \boldsymbol{\pi}=\mathbf{0})$, and the matrix $\boldsymbol{J}_{\pi}=\partial g(\boldsymbol{q}, \boldsymbol{\theta}, \boldsymbol{\pi}) / \partial \boldsymbol{\pi}$ is the manipulator Jacobian with respect to the geometrical parameters $\boldsymbol{\pi}$.

The above presented equation (3) is the basic expression for the robot calibration that allows the user to obtain the desired geometric and elastostatic parameters $\Delta \boldsymbol{\pi}$ and $\boldsymbol{k}_{\theta}$. For this purposes a set of experiments are carried out, in which the end-effector locations $\left\{\boldsymbol{t}_{i}\right\}$ are measured by an external device for several manipulator configurations defined by the vectors of the actuated coordinates $\left\{\boldsymbol{q}_{i}\right\}$. It is also assumed that the corresponding vectors of the external loading $\left\{\boldsymbol{F}_{i}\right\}$ are known. It is clear that corresponding system of linear equations can be solved for $\left(\Delta \boldsymbol{\pi}, \boldsymbol{k}_{\theta}\right)$ if the number of manipulator configurations $m$ is high enough and the vectors $\left\{\boldsymbol{q}_{i}, i=\overline{1, m}\right\}$ are different to ensure non-singularity of the relevant observation matrix used in the identification procedure. However, there are some difficulties here related to the estimation of the orientation components $\left\{\boldsymbol{\varphi}_{i}\right\}$ of the location vectors $\boldsymbol{t}_{i}=\left(\boldsymbol{p}_{i}, \boldsymbol{\varphi}_{i}\right)$. There are two main approaches that are considered below.

The usual approach is based on the straightforward utilization of equation (3), where each configuration $\boldsymbol{q}_{i}$ produces six scalar equations corresponding to the components of the sixdimensional location vector $\boldsymbol{t}_{i}=\left(p_{x i}, p_{y i}, p_{z i} \cdot \varphi_{x i} \cdot \varphi_{y i} \cdot \varphi_{z i}\right)^{T}$. Corresponding optimization problem allowing to compute the desired parameters $\left(\Delta \boldsymbol{\pi}, \boldsymbol{k}_{\theta}\right)$ can we written as follows

$$
\sum_{i=1}^{m}\left\|\boldsymbol{t}_{i}-\boldsymbol{g}_{0 i}-\boldsymbol{J}_{\pi i} \cdot \Delta \boldsymbol{\pi}-\boldsymbol{J}_{\theta \mathrm{i}} \cdot \boldsymbol{k}_{\theta} \cdot \boldsymbol{J}_{\theta \mathrm{i}}^{T} \cdot \boldsymbol{F}_{i}\right\|^{2} \rightarrow \min _{\Delta \boldsymbol{\pi}, \boldsymbol{k}_{\theta}}
$$

However, in practice, the orientation components $\left(\varphi_{x i} \cdot \varphi_{y i} \cdot \varphi_{z i}\right)$ cannot be measured directly, so these angles are computed using excessive number of measurements for the same configuration $\boldsymbol{q}_{i}$, which produce Cartesian coordinates $\left\{\left(p_{x i j}, p_{y i j}, p_{z i j}\right) \mid j=\overline{1, n} ; n \geq 3\right\}$ for several target points of the measurement tool attached to the manipulator mounting flange (Figure 1). Hence, instead of using $3 m n$ scalar equations, that can be theoretically obtained from the measurement data, this conventional approach uses only $6 m$ scalar equations for the identification. These may 


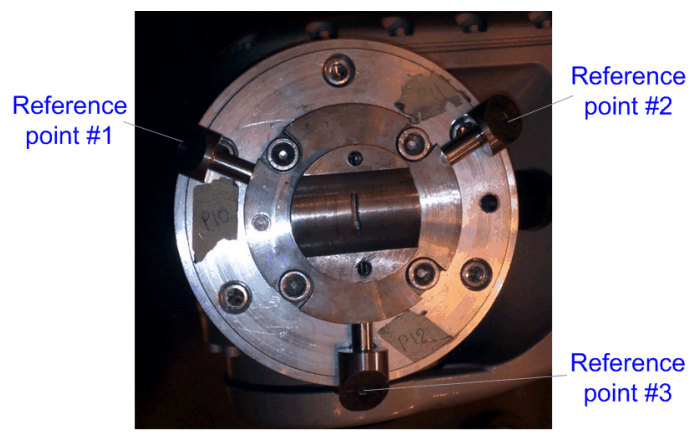

Figure 1. Typical measurement tool used for robot calibration.

obviously lead to some loss of the parameter estimation accuracy. Another difficulty is related to the definition of the vector norm in equation (4), where the six-dimensional residuals are not homogeneous. It is clear that the position and orientation components must be normalized before computing the squared sum, but it is a non-trivial step affecting the accuracy (in practice, the normalization factors are usually defined intuitively).

To overcome this difficulty it is proposed to reformulate the optimization problem (4) using only data directly available from the measurement system, i.e. the Cartesian coordinates of all reference points $\boldsymbol{p}_{i j}=\left(p_{x i j}, p_{y i j}, p_{z i j}\right)^{T}$ (Figure 1). This idea allows us to obtain homogeneous identification equations where each residual has the same unit ( $\mathrm{mm}$, for instance), and the optimization problem is rewritten as follows

$$
\sum_{i=1}^{m} \sum_{j=1}^{n}\left\|\boldsymbol{p}_{i j}-\boldsymbol{g}_{0 i j}^{(p)}-\boldsymbol{J}_{\pi i j}^{(p)} \cdot \Delta \boldsymbol{\pi}-\left[\boldsymbol{J}_{\theta \mathrm{ij}} \cdot \boldsymbol{k}_{\theta} \cdot \boldsymbol{J}_{\theta \mathrm{ij}}^{T} \cdot \boldsymbol{F}_{i}\right]^{(p)}\right\|^{2} \rightarrow \min _{\Delta \boldsymbol{\pi}, \boldsymbol{k}_{\theta}}
$$

Here, the superscripts "(p)" indicate the position components (three Cartesian coordinates) of the corresponding location vectors, the index " $i$ " defines the manipulator configuration number, while the index " $j$ " denotes the reference point number. An obvious advantage of this formulation is simplicity in the vector norm definition (conventional Euclidian norm can be applied here reasonably, the normalization is not required) and elimination of the problem of the weighting coefficient selection. In fact, under assumption that the measurement noise is presented as a set of i.i.d. random values (similar for all directions $\mathrm{x}, \mathrm{y}, \mathrm{z}$ and for all measurement configurations), the optimal linear estimator should have equal weights. Besides, there are some potential benefits in the identification accuracy here, since the total number of the scalar equations increases from $6 m$ to $3 m n$.

To prove advantages of the second approach based on the objective function (5), the following sections will be devoted to the development of the dedicated identification algorithm and the comparison study of the conventional and proposed techniques.

\section{Identification algorithm}

Let us assume that the measurement tool has $n$ reference points $(n \geq 3)$ that are used to estimate relevant vectors of the Cartesian coordinates $\boldsymbol{p}_{i}^{j}=\left(p_{x i}^{j}, p_{y i}^{j}, p_{z i}^{j}\right)^{T}$ for $m$ manipulator configurations. Using the homogeneous transformation technique, corresponding geometric model (1) can be presented as the matrix product

$$
\boldsymbol{T}_{i}^{j}=\boldsymbol{T}_{\text {base }} \cdot \boldsymbol{T}_{\text {robot }}\left(\boldsymbol{q}_{i}, \boldsymbol{\theta}_{i}, \boldsymbol{\pi}\right) \cdot \boldsymbol{T}_{\text {tool }}^{j}
$$

where the vectors $\boldsymbol{p}_{i}^{j}$ are incorporated in the forth column of $\boldsymbol{T}_{i}^{j}$, the matrix $\boldsymbol{T}_{\text {base }}$ defines the robot base location, the matrices $\boldsymbol{T}_{\text {tool }}^{j}, j=\overline{1, n}$ describe locations of the reference points that are 
observed by the measurement system (see Figure 1), and the matrix function $\boldsymbol{T}_{\text {robot }}\left(\boldsymbol{q}_{i}, \boldsymbol{\theta}_{i}, \boldsymbol{\pi}\right)$ describes the manipulator geometry and depends on the current values of the actuated coordinates $\boldsymbol{q}$, the robot elastostatic deflections $\boldsymbol{\theta}$, and the vector of the parameters $\boldsymbol{\pi}$ to be estimated.

Taking into account that any homogeneous transformation matrix $\boldsymbol{T}_{a}^{b}$ can be split into the rotational $\boldsymbol{R}_{a}^{b}$ and translational $\boldsymbol{p}_{a}^{b}$ components and presented as

$$
\boldsymbol{T}_{a}^{b}=\left[\begin{array}{cc}
\boldsymbol{R}_{a}^{b} & \boldsymbol{p}_{a}^{b} \\
\mathbf{0} & 1
\end{array}\right]
$$

the Cartesian coordinates of the reference points $\boldsymbol{p}_{i}^{j}, j=\overline{1, n}$ corresponding to the configuration $\boldsymbol{q}_{i}$ can be expressed in the following form

$$
\boldsymbol{p}_{i}^{j}=\boldsymbol{p}_{\text {base }}+\boldsymbol{R}_{\text {base }} \cdot \boldsymbol{p}_{\text {robot }}\left(\boldsymbol{q}_{i}, \boldsymbol{\theta}_{i}, \boldsymbol{\pi}\right)+\boldsymbol{R}_{\text {base }} \cdot \boldsymbol{R}_{\text {robot }}\left(\boldsymbol{q}_{i}, \boldsymbol{\theta}_{i}, \boldsymbol{\pi}\right) \cdot \boldsymbol{p}_{\text {tool }}^{j}
$$

This equation should be accompanied by the elastostatic model $\boldsymbol{\theta}_{i}=\boldsymbol{k}_{\theta} \cdot \boldsymbol{J}_{\theta}^{\mathrm{T}}\left(\boldsymbol{q}_{i}, \boldsymbol{\theta}_{i}, \boldsymbol{\pi}\right) \cdot \boldsymbol{F}_{i}$ that allows us to obtain $3 m n$ scalar equations for the identification purposes, where the following vectors/matrices are treated as unknowns: $\boldsymbol{p}_{\text {base }}, \boldsymbol{R}_{\text {base }}, \boldsymbol{p}_{\text {tool }}^{j}, \boldsymbol{k}_{\theta}$ and $\boldsymbol{\pi}$.

To simplify computations, it is proposed to split the identification procedure into two steps. The first one deals with the estimation of $\boldsymbol{p}_{\text {base }}, \boldsymbol{R}_{\text {base }}, \boldsymbol{p}_{\text {tool }}^{j}$, which are related to the base and tool transformations (assuming that the manipulator parameters are known). The second step focuses on the estimation of $\boldsymbol{k}_{\theta}$ and $\boldsymbol{\pi}$ under assumption that the base and tool components are already identified. To achieve desired accuracy, these steps are repeated iteratively several times.

Step 1. For the first step, taking into account that the errors in the base orientation are relatively small, the matrix $\boldsymbol{R}_{\text {base }}$ is presented in the following form

$$
\boldsymbol{R}_{\text {base }}=\left[\sim \varphi_{\text {base }}\right]+\boldsymbol{I}
$$

where $\boldsymbol{I}$ is $3 \times 3$ identity matrix, vector $\varphi_{\text {base }}$ includes the deviations in the base orientation, and the operator "[ ]" transforms the vector $\varphi=\left(\varphi_{x}, \varphi_{y}, \varphi_{z}\right)^{T}$ in the skew symmetric matrix as

$$
[\sim \varphi]=\left[\begin{array}{ccc}
0 & -\varphi_{z} & \varphi_{y} \\
\varphi_{z} & 0 & -\varphi_{x} \\
-\varphi_{y} & \varphi_{x} & 0
\end{array}\right]
$$

It should be mentioned that such linearization is valid only when the orientation of the base frame of the measurement system has been preliminarily specified close to the orientation of the robot base. This adjustment is usually executed via calibration of the measurement system using its embedded software and can be performed for the work-cell with both a single and several robots. This leads to the following presentation of equation (8)

$$
\boldsymbol{p}_{i}^{j}=\boldsymbol{p}_{\text {base }}+\boldsymbol{p}_{\text {robot }}^{i}-\left[\sim \boldsymbol{p}_{\text {robot }}^{i}\right] \cdot \boldsymbol{\varphi}_{\text {base }}+\boldsymbol{R}_{\text {base }} \cdot \boldsymbol{R}_{\text {robot }}\left(\boldsymbol{q}_{i}, \boldsymbol{\theta}_{i}, \boldsymbol{\pi}\right) \cdot \boldsymbol{p}_{\text {tool }}^{j}
$$

Further, taking into account (9) the last term of (11) can be presented as

$$
\boldsymbol{R}_{\text {base }} \cdot \boldsymbol{R}_{\text {robot }}\left(\boldsymbol{q}_{i}, \boldsymbol{\theta}_{i}, \boldsymbol{\pi}\right) \cdot \boldsymbol{p}_{\text {tool }}^{j}=\boldsymbol{R}_{\text {robot }}\left(\boldsymbol{q}_{i}, \boldsymbol{\theta}_{i}, \boldsymbol{\pi}\right) \cdot \boldsymbol{p}_{\text {tool }}^{j}+\boldsymbol{\varphi}_{\text {base }} \times\left(\boldsymbol{R}_{\text {robot }}\left(\boldsymbol{q}_{i}, \boldsymbol{\theta}_{i}, \boldsymbol{\pi}\right) \cdot \boldsymbol{p}_{\text {tool }}^{j}\right)
$$

where the symbol " $\times$ " denotes the vector product (it can be proved that for any vectors $\boldsymbol{\varphi}, \boldsymbol{p}$ it is true that $[\sim \varphi] \cdot \boldsymbol{p}=\boldsymbol{\varphi} \times \boldsymbol{p}$ ). In this case, the second term in equation (12) is the vector product of $\boldsymbol{\varphi}_{\text {base }}$ and $\left(\boldsymbol{R}_{\text {robot }}\left(\boldsymbol{q}_{i}, \boldsymbol{\theta}_{i}, \boldsymbol{\pi}\right) \cdot \boldsymbol{p}_{\text {tool }}^{j}\right)$, whose order influences on the sign before the term. This transformation yields the following expression $-\boldsymbol{R}_{\text {robot }}\left(\boldsymbol{q}_{i}, \boldsymbol{\theta}_{i}, \boldsymbol{\pi}\right) \cdot \boldsymbol{p}_{\text {tool }}^{j} \times \boldsymbol{\varphi}_{\text {base }}$ for the second term of equation (12), where the vectors $\boldsymbol{p}_{\text {tool }}^{j}$ and $\boldsymbol{\varphi}_{\text {base }}$ can be also rearranged (with 
changing the term sign for the second time). Further, after expensing the vector product in the matrix form, the latter can be rewritten as

$$
\boldsymbol{R}_{\text {base }} \cdot \boldsymbol{R}_{\text {robot }}\left(\boldsymbol{q}_{i}, \boldsymbol{\theta}_{i}, \boldsymbol{\pi}\right) \cdot \boldsymbol{p}_{\text {tool }}^{j}=\boldsymbol{R}_{\text {robot }}\left(\boldsymbol{q}_{i}, \boldsymbol{\theta}_{i}, \boldsymbol{\pi}\right) \cdot\left(\boldsymbol{I}+\left[\sim \boldsymbol{\varphi}_{\text {base }}\right]\right) \cdot \boldsymbol{p}_{\text {tool }}^{j}
$$

Finally, taking into account (9), one can get that for the considered problem (due to the specific structure of $\left.\boldsymbol{R}_{\text {base }}\right)$ the matrices $\boldsymbol{R}_{\text {base }}$ and $\boldsymbol{R}_{\text {robot }}\left(\boldsymbol{q}_{i}, \boldsymbol{\theta}_{i}, \boldsymbol{\pi}\right)$ are commutative and can be rearranged in expression (11), i.e.

$$
\boldsymbol{R}_{\text {base }} \cdot \boldsymbol{R}_{\text {robot }}\left(\boldsymbol{q}_{i}, \boldsymbol{\theta}_{i}, \boldsymbol{\pi}\right) \cdot \boldsymbol{p}_{\text {tool }}^{j}=\boldsymbol{R}_{\text {robot }}\left(\boldsymbol{q}_{i}, \boldsymbol{\theta}_{i}, \boldsymbol{\pi}\right) \cdot \boldsymbol{R}_{\text {base }} \cdot \boldsymbol{p}_{\text {tool }}^{j}
$$

So, expression (11) can be presented in the following form

$$
\boldsymbol{p}_{i}^{j}=\boldsymbol{p}_{\text {base }}+\boldsymbol{p}_{\text {robot }}^{i}-\left[\sim \boldsymbol{p}_{\text {robot }}^{i}\right] \cdot \boldsymbol{\varphi}_{\text {base }}+\boldsymbol{R}_{\text {robot }}^{i} \cdot \boldsymbol{u}_{\text {tool }}^{j}
$$

where

$$
\boldsymbol{u}_{\text {tool }}^{j}=\boldsymbol{R}_{\text {base }} \cdot \boldsymbol{p}_{\text {tool }}^{j}
$$

For the convenient purposes equation (15) can be rewritten in a matrix form as

$$
\boldsymbol{p}_{i}^{j}=\boldsymbol{p}_{\text {robot }}^{i}+\left[\begin{array}{lll}
\boldsymbol{I} & {\left[\begin{array}{ll}
\sim \boldsymbol{p}_{\text {robot }}^{i}
\end{array}\right]^{T}} & \boldsymbol{R}_{\text {robot }}^{i}
\end{array}\right]\left[\begin{array}{c}
\boldsymbol{p}_{\text {base }} \\
\boldsymbol{\varphi}_{\text {base }} \\
\boldsymbol{u}_{\text {tool }}^{j}
\end{array}\right]
$$

where $\boldsymbol{p}_{\text {robot }}^{i}$ and $\boldsymbol{R}_{\text {robot }}^{i}$ are defined as follows

$$
\boldsymbol{p}_{\text {robot }}^{i}=\boldsymbol{p}_{\text {robot }}\left(\boldsymbol{q}_{i}, \boldsymbol{\theta}_{i}, \boldsymbol{\pi}\right) ; \quad \boldsymbol{R}_{\text {robot }}^{i}=\boldsymbol{R}_{\text {robot }}\left(\boldsymbol{q}_{i}, \boldsymbol{\theta}_{i}, \boldsymbol{\pi}\right)
$$

Here the vectors $\boldsymbol{p}_{\text {base }}, \boldsymbol{\varphi}_{\text {base }}$ and $\boldsymbol{u}_{\text {tool }}^{j}, j=\overline{1, n}$ are treated as unknowns.

Applying to the linear system (17) the least-square technique, the desired vectors defining the base and tool transformations can be expressed as follows

$$
\left[\boldsymbol{p}_{\text {base }} ; \boldsymbol{\varphi}_{\text {base }} ; \boldsymbol{u}_{\text {tool }}^{1} ; \ldots \boldsymbol{u}_{\text {tool }}^{n}\right]=\left(\sum_{i=1}^{m} \boldsymbol{A}_{i}^{j^{T}} \boldsymbol{A}_{i}^{j}\right)^{-1}\left(\sum_{i=1}^{m} \boldsymbol{A}_{i}^{j^{T}} \Delta \boldsymbol{p}_{i}\right)
$$

where

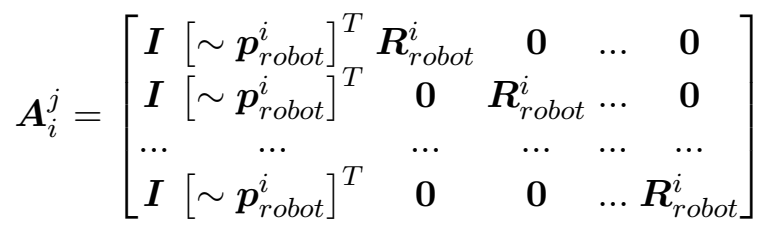

and the residuals are integrated in a single vector $\Delta \boldsymbol{p}_{i}=\left[\Delta \boldsymbol{p}_{i}^{1} ; \ldots \Delta \boldsymbol{p}_{i}^{n}\right]$. Finally, the variables defining the location to the reference points are computed using expression (16) as $\boldsymbol{p}_{\text {tool }}^{j}=$ $\boldsymbol{R}_{b a s e}^{T} \cdot \boldsymbol{u}_{\text {tool }}^{j}$. This allows us to find the homogeneous transformation matrices $\boldsymbol{T}_{b a s e}$ and $\boldsymbol{T}_{\text {tool }}^{j}$ that are contained in expression (6).

Step 2. On the second step, the remaining parameters $\boldsymbol{\pi}$ and $\boldsymbol{k}_{\theta}$, which define the manipulator geometry and the elastostatic properties, are estimated. For this purpose, the principal system 
(6) is linearized and rewritten in the form

$$
\boldsymbol{p}_{i}^{j}=\boldsymbol{p}_{\text {robot }}^{i}+\boldsymbol{J}_{\pi i}^{j(p)} \cdot \Delta \boldsymbol{\pi}+\boldsymbol{A}_{\theta i}^{j(p)} \cdot \chi
$$

where the subscript " $(p)$ " denotes the positional components (three first rows) of the corresponding matrices, $\Delta \pi$ is the vector of geometrical errors, $\chi$ is the vector of unknowns derived from the elements of the compliance matrix $\boldsymbol{k}_{\theta}$, the matrix $\boldsymbol{J}_{\pi i}^{j}$ is the geometric Jacobian computed for the configuration $\boldsymbol{q}_{i}$ with respect to the reference point $j$, and $\boldsymbol{A}_{\theta i}^{j(p)}$ is derived by relevant transformation of the last term of equation (3) into the vector form:

$$
\boldsymbol{A}_{i}^{j}=\left[\boldsymbol{J}_{1 i}^{j} \boldsymbol{J}_{1 i}^{j}{ }^{T} \boldsymbol{F}_{i}, \ldots, \boldsymbol{J}_{n i}^{j} \boldsymbol{J}_{n i}^{j}{ }^{T} \boldsymbol{F}_{i}\right]
$$

In the last expression, $\boldsymbol{J}_{1 i}^{j}, \ldots, \boldsymbol{J}_{n i}^{j}$ denote the vector-columns obtained by splitting of the geometric Jacobian $\boldsymbol{J}_{\pi i}^{j}$. For the computational convenience expression (21) can be presented in the matrix form

$$
\Delta \boldsymbol{p}_{i}^{j}=\left[\boldsymbol{J}_{i}^{j(p)}, \boldsymbol{A}_{i}^{j(p)}\right] \cdot\left[\begin{array}{c}
\Delta \boldsymbol{\pi} \\
\chi
\end{array}\right]
$$

where $\Delta \boldsymbol{p}_{i}^{j}=\boldsymbol{p}_{i}^{j}-\boldsymbol{p}_{\text {robot }}^{i}$ is the residual vector corresponding to the jth reference point for the ith manipulator configuration.

Applying to this system the least-square technique, the desired vectors $\Delta \pi, \boldsymbol{\chi}$, defining the manipulator geometric and elastostatic properties, can be expressed as

$$
\left[\begin{array}{c}
\Delta \boldsymbol{\pi} \\
\chi
\end{array}\right]=\left(\sum_{i=1}^{m} \sum_{j=1}^{n} \boldsymbol{B}_{i}^{j(p)^{T}} \boldsymbol{B}_{i}^{j(p)}\right)^{-1}\left(\sum_{i=1}^{m} \sum_{j=1}^{n} \boldsymbol{B}_{i}^{j(p)^{T}} \Delta \boldsymbol{p}_{i}^{j}\right)
$$

where $\boldsymbol{B}_{i}^{j(p)}=\left[\boldsymbol{J}_{i}^{j(p)}, \boldsymbol{A}_{i}^{j(p)}\right]$.

It should be noted that, to achieve the desired accuracy, the steps 1 and 2 should be repeated iteratively. This iterative procedure allows us also to overcome decrease of the identification accuracy due to linearization since on each iteration the model linearization takes into account parameters identified on the previous step.

\section{Comparison analysis}

To illustrate the efficiency of the proposed technique, let us compare its accuracy with the conventional one that operates with the full pose information. Their distinctions and particularities can be described as follows:

- Approach \#1 (conventional): The identification is based on the full pose information $\boldsymbol{t}_{0}=\left[\boldsymbol{p}_{0}, \boldsymbol{\varphi}_{0}\right]$, where both position $\boldsymbol{p}_{0}$ and orientation $\boldsymbol{\varphi}_{0}$ vectors are computed from the Cartesian coordinates of three reference points $\left\{\boldsymbol{p}_{1}, \boldsymbol{p}_{2}, \boldsymbol{p}_{3}\right\}$ located on the manipulator end-effector (see Figure 1).

- Approach \#2 (proposed): The identification is based on the partial pose information, where three measurement points $\left\{\boldsymbol{p}_{1}, \boldsymbol{p}_{2}, \boldsymbol{p}_{3}\right\}$ are directly included in the objective function to be minimized by the identification algorithm. 


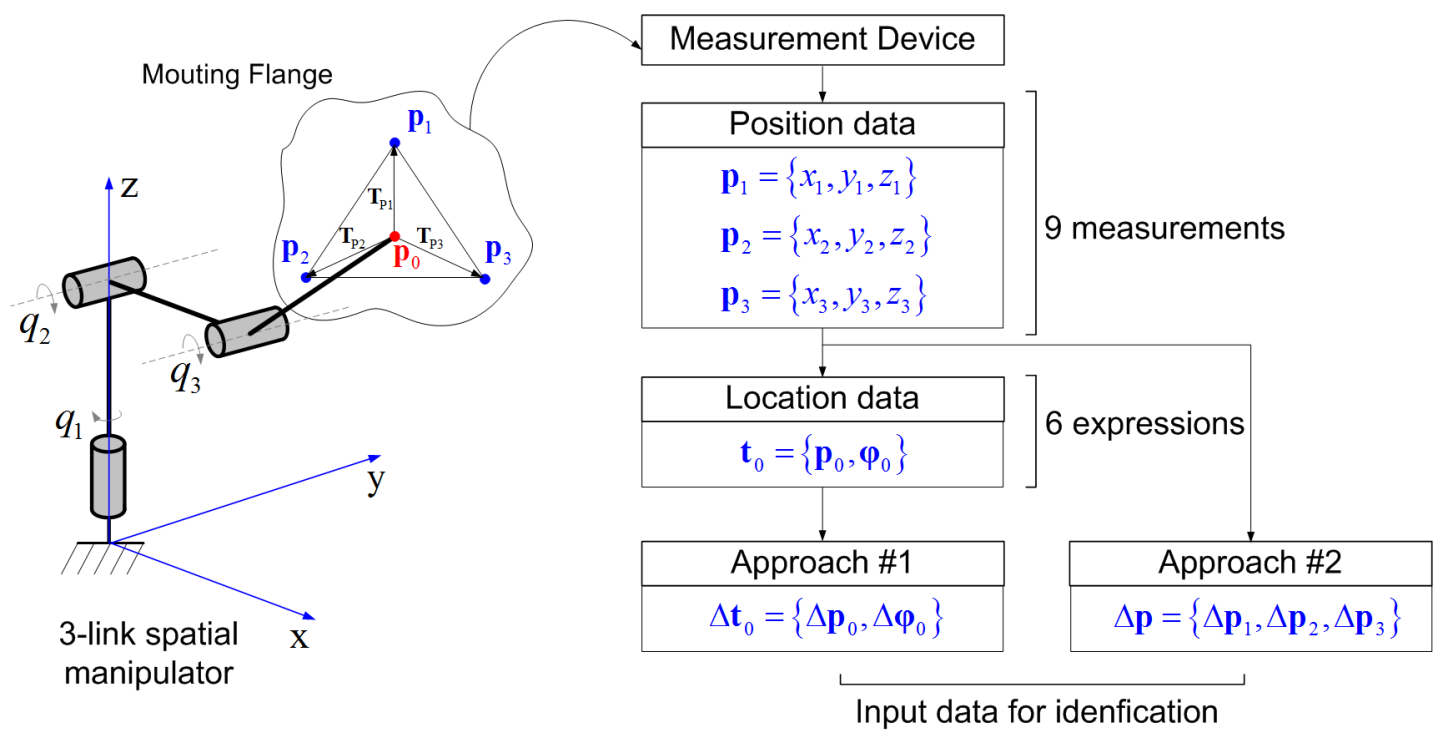

Figure 2. Input data for two identification approaches.

Let us consider a 3 d.o.f. serial manipulator whose geometry is described by the following equations

$$
\begin{aligned}
& x=\left(l_{2} \cos q_{2}+l_{3} \cos \left(q_{2}+q_{3}\right)\right) \cos q_{1} \\
& y=\left(l_{2} \cos q_{2}+l_{3} \cos \left(q_{2}+q_{3}\right)\right) \sin q_{1} \\
& z=l_{1}+l_{2} \sin q_{2}+l_{3} \sin \left(q_{2}+q_{3}\right) \\
& \varphi_{x}=0 ; \quad \varphi_{y}=q_{2}+q_{3} ; \quad \varphi_{z}=q_{1}
\end{aligned}
$$

where $q_{1}, q_{2}, q_{3}$ are the actuator coordinates and $l_{1}, l_{2}, l_{3}$ are the link lengths to be identified. The manipulator kinematics and procedure of the input data preparation for the approaches \#1 and \#2 are presented in Figure 2.

For simulation study, the following manipulator parameters have been assigned: $l_{1}=1 \mathrm{~m}, l_{2}=$ $0.8 \mathrm{~m}, l_{3}=0.6 \mathrm{~m}$. It was assumed that the geometric errors in the link lengths and the actuated joint offsets are respectively $\Delta l_{1}=3.0 \mathrm{~mm}, \Delta l_{2}=2.0 \mathrm{~mm}, \Delta l_{3}=5.0 \mathrm{~mm}$ and $\Delta q_{1}=1.0 \mathrm{deg}$, $\Delta q_{2}=0.5 \mathrm{deg}, \Delta q_{3}=2.0 \mathrm{deg}$. Besides, it was also assumed that the measurements errors are i.i.d. random variables with the standard deviation $\sigma=0.01 \mathrm{~mm}$ (which perfectly corresponds to the precision of the equipment used for the experimental validations). The desired parameters were estimated using three measurement configurations, which were generated randomly. To obtain reliable statistics, the simulation of the calibration experiments have been repeated 1000 times.

Simulation results are summarized in Table 1, which presents the standard deviations for the estimates of the desired parameters (corresponding mean values of the estimates are equal to the assigned values with the high accuracy). Here, the improvement factor defines the ratio between the parameters identification accuracy using the conventional full-pose measurements and the proposed enhanced partial pose measurements. As follows from these results, the proposed approach ensures the accuracy improvement in the estimation of the link length deviations $\Delta l_{i}$ by the factor of $2.25 \ldots 3.83$, while the accuracy improvement for the joint offsets estimations $\Delta q_{i}$ is slightly less, up to 3.33. This confirms advantages of the proposed approach, but it should be mentioned that these numbers are obtained for particular set of the measurement configurations and particular normalization factor utilized in the approach \#1. However, as follows from our study, approach \#2 always provides essentially better results. 
Table 1. Identification accuracy for different identification approaches based on 3 measurement configurations.

\begin{tabular}{cccc}
\hline \multirow{2}{*}{ Parameter } & \multicolumn{2}{c}{ Standard deviation } & \\
\cline { 2 - 3 } & Approach \#1 & Approach \#2 & \\
\hline$\Delta l_{1}$ & $0.069 \mathrm{~mm}$ & $0.018 \mathrm{~mm}$ & 3.83 \\
$\Delta l_{2}$ & $0.019 \mathrm{~mm}$ & $0.006 \mathrm{~mm}$ & 3.17 \\
$\Delta l_{3}$ & $0.009 \mathrm{~mm}$ & $0.004 \mathrm{~mm}$ & 2.25 \\
$\Delta q_{1}$ & $0.187 \mathrm{mdeg}$ & $0.185 \mathrm{mdeg}$ & 1.01 \\
$\Delta q_{2}$ & $3.742 \mathrm{mdeg}$ & $1.123 \mathrm{mdeg}$ & 3.33 \\
$\Delta q_{3}$ & $1.432 \mathrm{mdeg}$ & $0.866 \mathrm{mdeg}$ & 1.65 \\
\hline
\end{tabular}

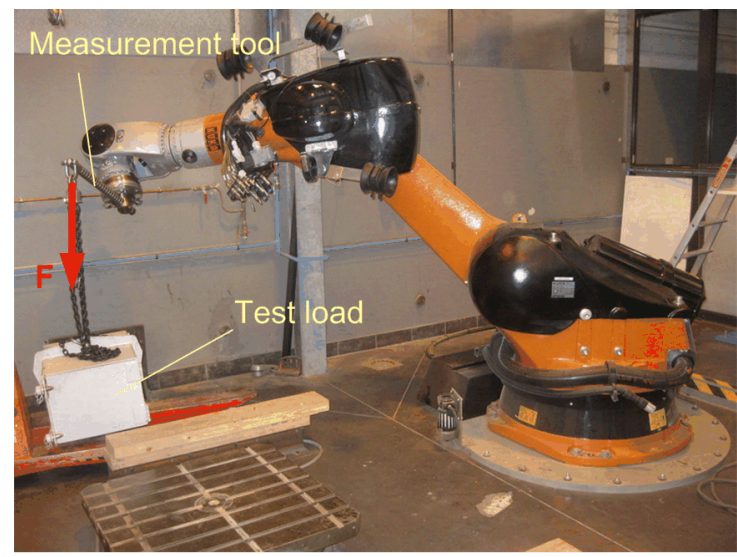

Figure 3. Experimental setup for the identification of the elastostatic parameters.

\section{Application example}

To confirm the applicability of the proposed calibration techniques and to demonstrate their benefits from engineering point of view, this Section presents the identification results for geometric and elastostatic calibration of the industrial robot KUKA KR-270 (see Figure 3).

The complete and irreducible geometric model for the considered manipulator includes 18 principle parameters , 6 parameters describing the laser tracker location with respect to the robot base (both position and orientation) and 9 parameters describing locations of the endeffector reference points with respect to the manipulator mounting flange (positions only for three points). The optimal measurement configurations have been generated using the design of experiments technique [33], whose objective function takes into account the work-cell constraints and the manipulator joint limits. For the experimental study 18 measurement configurations have been selected, in which reference point locations have been measured several times. It should be mentioned that, to insure visibility of all reference points, two laser tracker locations have been used. This increases the number of parameters to be identified by 6 (an additional base transformation for the second measurement system location).

Using the obtained measurement data, the two-step identification procedure has been applied. On the first step, the base and tool transformations have been computed, corresponding results are presented in Table 2. Here, the vectors $\boldsymbol{p}_{\text {base }}^{i}, \boldsymbol{\varphi}_{\text {base }}^{i}, i=\overline{1,2}$ define required modification in the position and orientation of the ith base frame (each of which corresponds to the different laser-tracker location used in the experimental study); the vectors $\boldsymbol{p}_{\text {tool }}^{j}$ describe the tool parameters for the jth reference point $(j=\overline{1,3})$. On the second step, these transformations have been used for the identification of the manipulator geometric parameters, which are presented in Table 3. In this table, the parameters of the "complete, continues and irreducible" geometrical model are listed in the first column (from the base to tool order). For some of these parameters, corresponding deviations in DH-representation are given, where the symbol $p$ denotes translational parameters; $\varphi$ is the rotational parameter; $\Delta q_{i}$ denotes the offsets in the actuated joints, the subscript indicates the translation/rotation axis and the coordinate frame number. In the 
Table 2. Identification results for manipulator base and tool transformations (for geometric calibration).

\begin{tabular}{ccccccc}
\hline $\mathbf{p}_{\text {base }}^{1}, \mathrm{~mm}$ & $\varphi_{\text {base }}^{1}, \mathrm{mrad}$ & $\mathbf{p}_{\text {base }}^{2}, \mathrm{~mm}$ & $\varphi_{\text {base }}^{2}, \mathrm{mrad}$ & $\mathbf{p}_{\text {tool }}^{1}, \mathrm{~mm}$ & $\mathbf{p}_{\text {tool }}^{2}, \mathrm{~mm}$ & $\mathbf{p}_{\text {tool }}^{3}, \mathrm{~mm}$ \\
\hline$\left[\begin{array}{c}-0.02 \\
0.01 \\
0.06\end{array}\right]$ & {$\left[\begin{array}{c}-0.07 \\
0.02 \\
-0.29\end{array}\right]$} & {$\left[\begin{array}{l}-0.10 \\
-0.10 \\
-0.08\end{array}\right]$} & {$\left[\begin{array}{c}-0.15 \\
0.10 \\
-0.27\end{array}\right]$} & {$\left[\begin{array}{c}277.23 \\
-46.53 \\
-93.87\end{array}\right]$} & {$\left[\begin{array}{c}276.49 \\
-48.25 \\
94.05\end{array}\right]$} & {$\left[\begin{array}{c}278.44 \\
103.73 \\
-2.17\end{array}\right]$} \\
\hline
\end{tabular}

Table 3. Identification results for manipulator geometric parameters.

\begin{tabular}{cccc}
\hline Parameter & Unit & Value & CI \\
\hline$p_{x 1} \equiv \Delta d_{2}$ & {$[\mathrm{~mm}]$} & -0.353 & 0.086 \\
$p_{y 1}$ & {$[\mathrm{~mm}]$} & 0.426 & 0.272 \\
$\varphi_{x 1}$ & {$[\mathrm{deg}]$} & 0.015 & 0.005 \\
$\Delta q_{2}$ & {$[\mathrm{deg}]$} & -0.007 & 0.005 \\
$p_{x 2} \equiv \Delta d_{3}$ & {$[\mathrm{~mm}]$} & 0.458 & 0.082 \\
$\varphi_{x 2}$ & {$[\mathrm{deg}]$} & 0.022 & 0.014 \\
$\varphi_{z 2}$ & {$[\mathrm{deg}]$} & -0.023 & 0.005 \\
$\Delta q_{3}$ & {$[\mathrm{deg}]$} & -0.023 & 0.019 \\
$p_{x 3} \equiv \Delta d_{4}$ & {$[\mathrm{~mm}]$} & -0.214 & 0.089 \\
$p_{z 3} \equiv \Delta d_{5}$ & {$[\mathrm{~mm}]$} & -0.508 & 0.363 \\
$\varphi_{z 3}$ & {$[\mathrm{deg}]$} & -0.011 & 0.017 \\
$\Delta q_{4}$ & {$[\mathrm{deg}]$} & 0.001 & 0.008 \\
$p_{y 4}$ & {$[\mathrm{~mm}]$} & -0.167 & 0.113 \\
$p_{z 4}$ & {$[\mathrm{~mm}]$} & -0.018 & 0.073 \\
$\varphi_{z 4}$ & {$[\mathrm{deg}]$} & 0.025 & 0.015 \\
$\Delta q_{5}$ & {$[\mathrm{deg}]$} & -0.011 & 0.027 \\
$p_{z 5}$ & {$[\mathrm{~mm}]$} & 0.016 & 0.104 \\
$\varphi_{z 5}$ & {$[\mathrm{deg}]$} & -0.008 & 0.018 \\
\hline
\end{tabular}

third column of the table, the identified value are presented for which the confidence intervals are given in the forth column. It should be mentioned that in order to increase the identification accuracy, this two-step procedure has been repeated iteratively (for the presented case study, with the stopping criterion $10^{-7} \mathrm{~mm}$ for the difference in the positioning accuracy between two consecutive iterations, 280 steps have been required).

The above presented table includes 18 parameters, some of which cannot be modified in the robot control software. So, it is interesting to examine the effect of reducing the number of these parameters by setting them to their nominal values. Relevant analysis shows that the manipulator end-effector positioning error impact (because of such simplification) essentially differs from parameter to parameter, and they can be split into the following groups:

- Parameters $\left\{p_{x 1}, p_{y 1}, p_{x 2}, p_{x 3}, p_{z 3}, p_{y 4}, \Delta q_{2}, \Delta q_{3}, \varphi_{z 2}, \varphi_{z 3}, \varphi_{z 4}\right\}$, for which the loss of accuracy caused by neglecting varies from $0.10 \mathrm{~mm}$ to $0.95 \mathrm{~mm}$;

- Parameters $\left\{p_{z 4}, \Delta q_{5}, \varphi_{x 1}, \varphi_{x 2}, \varphi_{z 5}\right\}$, for which the loss of accuracy caused by neglecting varies from $0.03 \mathrm{~mm}$ to $0.08 \mathrm{~mm}$;

- Parameters $\left\{p_{z 5}, \Delta q_{4}\right\}$, for which the loss of accuracy caused by neglecting varies from $0.003 \mathrm{~mm}$ to $0.007 \mathrm{~mm}$.

Comparing to the milling accuracy required for the considered technological process, the above listed positioning error impacts are not negligible for the most of the geometric parameters. So, their deviations should be compensated either in geometric model embedded in the robot controller or on the step of generation of the machining trajectory.

The developed identification technique has been also applied to the elastostatic calibration. To take into account the influence of the gravity compensator (which creates the closed-loop) and to apply the virtual joint modeling approach developed for strictly serial robot [33, 34], an equivalent non-linear virtual spring is used (its stiffness depends on the joint variable $q_{2}$ ). However, to implement this idea, it is reasonable to consider a set of the compliance coefficients $\left\{\chi_{2 i}, i=1,2, \ldots\right\}$ corresponding to a number of different joint angles $\left\{q_{2 i}, i=1,2, \ldots\right\}$ that cover relevant joint limits (see [35] for more details on this approach). This yields the extended set of the elastostatic parameters $\chi_{e}=\left[\left(\chi_{21}, \chi_{22} \ldots\right), \chi_{3}, \ldots, \chi_{6}\right]$ to be identified and leads to the linear 


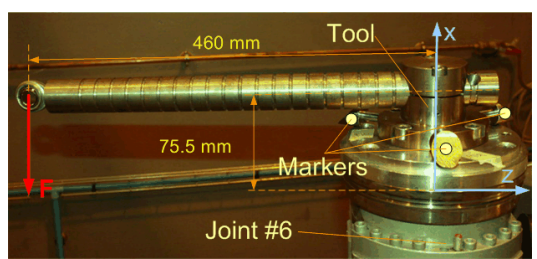

Figure 4. Measurement tool with three markers used for elastostatic calibration experiments.

Table 4. Identification results for base and tool transformations .

\begin{tabular}{ccccc}
\hline $\mathbf{p}_{\text {base }}, \mathrm{mm}$ & $\varphi_{\text {base }}, \mathrm{mrad}$ & $\mathbf{p}_{\text {tool }}^{1}, \mathrm{~mm}$ & $\mathbf{p}_{\text {tool }}^{2}, \mathrm{~mm}$ & $\mathbf{p}_{\text {tool }}^{3}, \mathrm{~mm}$ \\
\hline$\left[\begin{array}{c}0.27 \\
-4.83 \\
-3.73\end{array}\right]$ & {$\left[\begin{array}{c}5.34 \\
0.49 \\
-1.46\end{array}\right]$} & {$\left[\begin{array}{c}279.49 \\
-46.01 \\
-94.25\end{array}\right]$} & {$\left[\begin{array}{c}279.45 \\
-44.75 \\
93.64\end{array}\right]$} & {$\left[\begin{array}{c}280.37 \\
105.68 \\
-4.90\end{array}\right]$} \\
\hline
\end{tabular}

Table 5. Identified values of manipulator elastostatic parameters.

\begin{tabular}{cc}
\hline$\chi_{i}$ & Values, $[\mathrm{rad} / \mathrm{Nmm}]$ \\
\hline$\chi_{21}$ & 0.2870 .0003 \\
$\chi_{22}$ & 0.2770 .0004 \\
$\chi_{23}$ & 0.3020 .0005 \\
$\chi_{24}$ & 0.2930 .0010 \\
$\chi_{25}$ & 0.2460 .0007 \\
$\chi_{3}$ & 0.4160 .0011 \\
$\chi_{4}$ & 2.7860 .0071 \\
$\chi_{5}$ & 3.4830 .0120 \\
$\chi_{6}$ & 2.0740 .0267 \\
\hline
\end{tabular}

system of the identification equations similar to those considered in Section 3.

To find the measurement configurations that ensure the best identification accuracy, the design of experiments technique has been applied, which is based on the dedicated industry-oriented performance measure proposed in our previous work [30]. This yielded 15 optimal measurement configurations with five different angles $q_{2}$ that are distributed between the joint limits almost uniformly. These optimal configurations have been obtained taking into account physical constraints that are related to the joint limits and the possibility to apply the gravity force (work-cell obstacles and safety reasons).

At the measurement step, the manipulator was sequentially moved from one configuration to another, where the external loading $250 \mathrm{~kg}$ was applied to the special end-effector presented in Figure 4 (it allowed us to generate both external forces and torques). Corresponding experiment setup is shown in Figure 3. To measure the reference point Cartesian coordinates, the laser tracker system Leica AT901 was used. To evaluate manipulator elastostatic deflections, the reference point coordinates have been measured twice, before and after application of the external loading.

Using these measurement data, the two-step identification procedure has been applied. On the first step, the tool and base transformations have been computed; corresponding results are presented in Table 4. This table is organised similar to Table 2 for geometrical calibration, the only difference is related to the number of the laser-tracker locations used in the experimental study. On the second step, they have been used for the identification of the elastostatic parameters, which are presented in Table 5. It includes an extended set of the elastostatic parameters (compliances for the joints \#3-\#6 and equivalent compliances for the second joint, which take into account the gravity compensator impact).

Let us now illustrate the efficiency of the elastostatic calibration and related compliance error compensation technique by applying it to the robotic-based machining using the industrial robot KUKA KR-270. In accordance with the considered specifications, the technological process should be performed in the square area of the size $2000 \mathrm{~mm} 2000 \mathrm{~mm}$ located at the height 500 


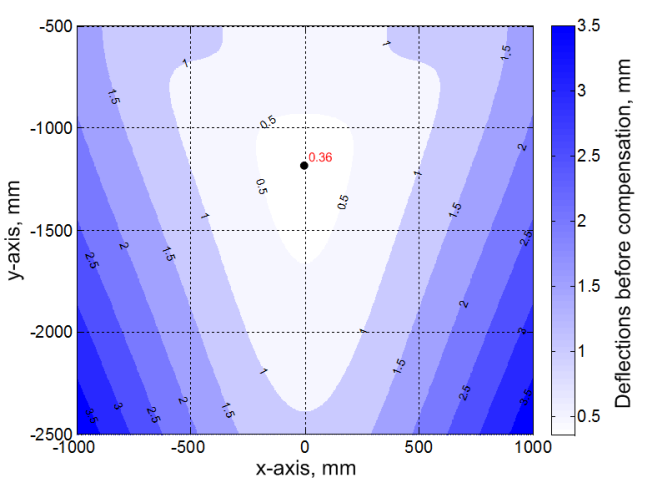

(a) Robot positioning accuracy in milling without compliance error compensation

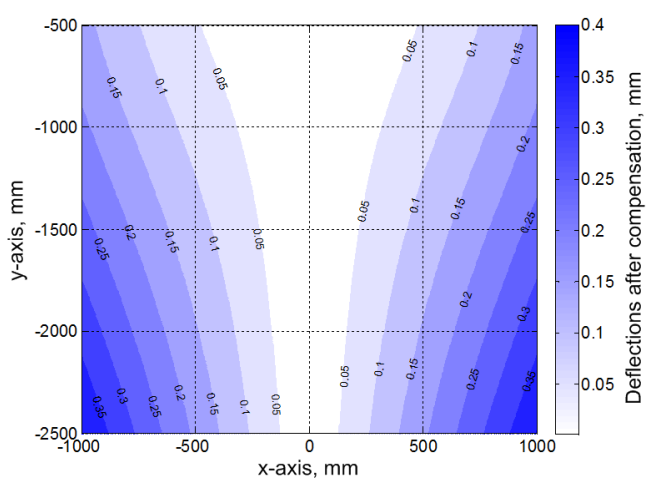

(b) Robot positioning accuracy in milling with compliance error compensation

Figure 5. Robot positioning accuracy before and after elastostatic calibration.

$\mathrm{mm}$ over the floor level. For comparison purpose, it is assumed that machining force is constant throughout the working area and it is equal to $\boldsymbol{F}=(0,360 N, 560 N, 0,0,0)^{T}$, which corresponds to a typical milling process. Relevant computations have been done throughout the required working area, where the end-effector deflections have been evaluated for the machining force $\boldsymbol{F}$. The computational results are summarized in Figure 5a, where the end-effector compliance errors are presented. These results show that the compliance errors are not negligible here and vary from 0.36 to $3.5 \mathrm{~mm}$. So, to achieve the desired precision, it is reasonable to apply the error compensation technique, which is based on the developed stiffness model. As a result, the robot positioning accuracy has been increased essentially (Figure 5b): the positioning errors are about $0.05 \mathrm{~mm}$ in the middle of workspace and are less that $0.4 \mathrm{~mm}$ at the borders. These results show that the obtained elastostatic model allows us to achieve essential improvement of the robot precision; it is able to compensate about $90 \%$ of elastostatic deflections caused by the machining force. Hence, such a model can be used for the compliance error compensation for the robotic based milling in industry.

\section{Conclusions}

The paper presents the advanced robot calibration technique that is based on the partial pose information, without explicit computation of the end-effector orientation. In contrast to previous works, the proposed technique utilizes the Cartesian coordinates measurements only, but several reference points of the end-effector are used. The proposed approach allows us to avoid the problem of non-homogeneity of the least-square objective, which arises in the conventional identification technique, where the full-pose information (both position and orientation) is considered. The technique does not require any normalization of the residuals and can be efficiently applied to both geometric and elastostatic calibration. As follows from the simulation analysis presented in the paper, the developed technique essentially improves the identification accuracy compared to the conventional one. The obtained theoretical results have been successfully applied for the geometric and elastostatic parameters identification of an industrial robot used in aerospace industry.

\section{Acknowledgements}

The work presented in this paper was partially funded by the ANR (Project ANR-2010-SEGI003-02-COROUSSO), France and FEDER ROBOTEX, France. 


\section{References}

[1] Hollerbach JM. The robotics review 1. 1989;:207-242.

[2] Mooring B, Driels M, Roth Z. Fundamentals of manipulator calibration. New York, NY, USA: John Wiley \& Sons, Inc.. 1991.

[3] Stone HW. Kinematic modeling, identification, and control of robotic manipulators. Vol. 29. Springer. 1987.

[4] Everett LJ, Hsu TW. The theory of kinematic parameter identification for industrial robots. Journal of dynamic systems, measurement, and control. 1988;110(1):96-100.

[5] Schröer K, Albright SL, Grethlein M. Complete, minimal and model-continuous kinematic models for robot calibration. Robotics and Computer-Integrated Manufacturing. 1997;13(1):73-85.

[6] Kikuchi H, Maeda Y, Sugi M, Arai T. Automatic calibration of assembly robots for instantaneous installation'plug \& produce'. Advanced Robotics. 2003;17(1):41-52.

[7] Meggiolaro MA, Dubowsky S, Mavroidis C. Geometric and elastic error calibration of a high accuracy patient positioning system. Mechanism and Machine Theory. 2005;40(4):415-427.

[8] Meng Y, Zhuang H. Autonomous robot calibration using vision technology. Robotics and ComputerIntegrated Manufacturing. 2007;23(4):436-446.

[9] Hollerbach J, Khalil W, Gautier M. Model identification. Springer Handbook of Robotics. 2008; :321-344.

[10] Verscheure D, Sharf I, Bruyninckx H, Swevers J, De Schutter J. Identification of contact dynamics parameters for stiff robotic payloads. Robotics, IEEE Transactions on. 2009;25(2):240-252.

[11] Nubiola A, Bonev IA. Absolute calibration of an abb irb 1600 robot using a laser tracker. Robotics and Computer-Integrated Manufacturing. 2013;29(1):236-245.

[12] Ji J, Sun L, Zhu Y. A novel high-speed and high-accuracy manipulator of planar five-link structure: Modeling and calibration. Advanced Robotics. 2009;23(1-2):89-112.

[13] Sun Y, Hollerbach JM. Active robot calibration algorithm. In: Icra. 2008. p. 1276-1281.

[14] Khalil W, Gautier M, Enguehard C. Identifiable parameters and optimum configurations for robots calibration. Robotica. 1991;9(01):63-70.

[15] Borm JH, Meng CH. Determination of optimal measurement configurations for robot calibration based on observability measure. The International Journal of Robotics Research. 1991;10(1):51-63.

[16] Daney D. Optimal measurement configurations for gough platform calibration. In: Robotics and automation, 2002. proceedings. icra'02. ieee international conference on. Vol. 1. IEEE. 2002. p. 147152.

[17] Klimchik A, Wu Y, Caro S, Pashkevich A. Design of experiments for calibration of planar anthropomorphic manipulators. arXiv preprint arXiv:12120511. 2012;.

[18] Driels MR, Pathre US. Significance of observation strategy on the design of robot calibration experiments. Journal of Robotic Systems. 1990;7(2):197-223.

[19] Ikits M, Hollerbach JM. Kinematic calibration using a plane constraint. In: Robotics and automation, 1997. proceedings., 1997 ieee international conference on. Vol. 4. IEEE. 1997. p. 3191-3196.

[20] He R, Zhao Y, Yang S, Yang S. Kinematic-parameter identification for serial-robot calibration based on poe formula. Robotics, IEEE Transactions on. 2010;26(3):411-423.

[21] Lightcap C, Hamner S, Schmitz T, Banks S. Improved positioning accuracy of the pa10-6ce robot with geometric and flexibility calibration. IEEE Transactions on Robotics. 2008;24(2):452-456.

[22] Zhuang H, Masory O, Yan J. Kinematic calibration of a stewart platform using pose measurements obtained by a single theodolite. In: Intelligent robots and systems 95.'human robot interaction and cooperative robots', proceedings. 1995 ieee/rsj international conference on. Vol. 2. IEEE. 1995. p. 329-334.

[23] Driels MR, Swayze WE. Automated partial pose measurement system for manipulator calibration experiments. Robotics and Automation, IEEE Transactions on. 1994;10(4):430-440.

[24] Bai Y, Zhuang H, Roth ZS. Experiment study of puma robot calibration using a laser tracking system. In: Soft computing in industrial applications, 2003. smcia/03. proceedings of the 2003 ieee international workshop on. IEEE. 2003. p. 139-144.

[25] Driels MR, Swayze LW, Potter LS. Full-pose calibration of a robot manipulator using a coordinatemeasuring machine. The International Journal of Advanced Manufacturing Technology. 1993;8(1):3441.

[26] Goswami A, Quaid A, Peshkin M. Complete parameter identification of a robot from partial pose information. In: Robotics and automation, 1993. proceedings., 1993 ieee international conference on. 
IEEE. 1993. p. 168-173.

[27] Joubair A, Slamani M, Bonev IA. Kinematic calibration of a five-bar planar parallel robot using all working modes. Robotics and Computer-Integrated Manufacturing. 2013;29(4):15-25.

[28] Abtahi M, Pendar H, Alasty A, Vossoughi G. Experimental kinematic calibration of parallel manipulators using a relative position error measurement system. Robotics and Computer-Integrated Manufacturing. 2010;26(6):799-804.

[29] Dumas C, Caro S, Che?rif M, Garnier S, Furet B. A methodology for joint stiffness identification of serial robots. In: Intelligent robots and systems (iros), 2010 ieee/rsj international conference on. IEEE. 2010. p. 464-469.

[30] Klimchik A, Wu Y, Pashkevich A, Caro S, Furet B. Optimal selection of measurement configurations for stiffness model calibration of anthropomorphic manipulators. Applied Mechanics and Materials. 2012;162:161-170.

[31] Klimchik A, Pashkevich A, Chablat D, Hovland G. Compliance error compensation technique for parallel robots composed of non-perfect serial chains. Robotics and Computer-Integrated Manufacturing. 2013;29(2):385-393.

[32] Pashkevich A, Klimchik A, Chablat D. Enhanced stiffness modeling of manipulators with passive joints. Mechanism and machine theory. 2011;46(5):662-679.

[33] Klimchik A, Bondarenko D, Pashkevich A, Briot S, Furet B. Compliance error compensation in robotic-based milling. In: Informatics in control, automation and robotics. Springer. 2014. p. 197216.

[34] Klimchik A, Chablat D, Pashkevich A. Stiffness modeling for perfect and non-perfect parallel manipulators under internal and external loadings. Mechanism and Machine Theory. 2014;79:1-28.

[35] Klimchik A, Wu Y, Dumas C, Caro S, Furet B, Pashkevich A. Identification of geometrical and elastostatic parameters of heavy industrial robots. In: Robotics and automation (icra), 2013 ieee international conference on. IEEE. 2013. p. 3707-3714. 